\title{
Distinct Roles of GABAergic Interneurons in the Regulation of Striatal Output Pathways
}

\author{
Aryn H. Gittis, ${ }^{1}$ Alexandra B. Nelson, ${ }^{3}$ Myo T. Thwin, ${ }^{1}$ Jorge J. Palop, ${ }^{1}$ and Anatol C. Kreitzer ${ }^{1,2,3}$ \\ ${ }^{1}$ Gladstone Institute of Neurological Disease, ${ }^{2}$ Department of Physiology, and ${ }^{3}$ Department of Neurology, University of California, San Francisco, \\ San Francisco, California 94158
}

\begin{abstract}
Striatal GABAergic microcircuits are critical for motor function, yet their properties remain enigmatic due to difficulties in targeting striatal interneurons for electrophysiological analysis. Here, we use Lhx6-GFP transgenic mice to identify GABAergic interneurons and investigate their regulation of striatal direct- and indirect-pathway medium spiny neurons (MSNs). We find that the two major interneuron populations, persistent low-threshold spiking (PLTS) and fast spiking (FS) interneurons, differ substantially in their excitatory inputs and inhibitory outputs. Excitatory synaptic currents recorded from PLTS interneurons are characterized by a small, nonrectifying AMPA receptor-mediated component and a NMDA receptor-mediated component. In contrast, glutamatergic synaptic currents in FS interneurons have a large, strongly rectifying AMPA receptor-mediated component, but no detectable NMDA receptor-mediated responses. Consistent with their axonal morphology, the output of individual PLTS interneurons is relatively weak and sparse, whereas FS interneurons are robustly connected to MSNs and other FS interneurons and appear to mediate the bulk of feedforward inhibition. Synaptic depression of FS outputs is relatively insensitive to firing frequency, and dynamic-clamp experiments reveal that these shortterm dynamics enable feedforward inhibition to remain efficacious across a broad frequency range. Surprisingly, we find that FS interneurons preferentially target direct-pathway MSNs over indirect-pathway MSNs, suggesting a potential mechanism for rapid pathway-specific regulation of striatal output pathways.
\end{abstract}

\section{Introduction}

The striatum is the main input nucleus of the basal ganglia and transforms converging thalamic and cortical inputs into two output streams, called the direct and the indirect pathways. Although the actions of these two pathways are complex, a simplified model of the basal ganglia circuit posits that the direct pathway facilitates directed movements while the indirect pathway terminates or suppresses movements (Smith et al., 1998). Imbalances in the activity of these two pathways are hypothesized to underlie numerous movement disorders, including Parkinson disease, Huntington's disease, and dystonia (Graybiel et al., 2000; Galvan and Wichmann, 2007).

At the neuronal level, initial activation of the direct and indirect pathways is determined by the firing of distinct classes of MSNs, which represent the sole output neurons of the striatum (Kreitzer, 2009). MSNs that project in the direct pathway express dopamine D1 receptors and send axons to the substantia nigra pars reticulata $(\mathrm{SNr})$. MSNs that project in the indirect pathway

Received Sept. 30, 2009; revised Dec. 21, 2009; accepted Dec. 30, 2009.

This work was supported by National Institutes of Health Grant R01 NS064984, the Pew Biomedical Scholars Program, the W.M. Keck Foundation, and the Wayne and Gladys Valley Foundation. We are grateful to M. A. Xu-Friedman for dynamic clamp and acquisition code for Igor Pro, and John Rubenstein and Inma Cobos for helpful discussion. We thank Nicole Calakos for her generous gift of the Drd1a-tdTomato mice and Sebastian Peck for help with microscopy. Some data in this study were acquired at the Nikon Imaging Center at the University of California, San Francisco.

Correspondence should be addressed to Anatol C. Kreitzer, Gladstone Institute of Neurological Disease, 1650 Owens Street, San Francisco, CA 94158. E-mail: akreitzer@gladstone.ucsf.edu.

D0I:10.1523/JNEUROSCI.4870-09.2010

Copyright $\odot 2010$ the authors $\quad 0270-6474 / 10 / 302223-12 \$ 15.00 / 0$ express dopamine D2 receptors and send axons to the globus pallidus (GP). Both classes of MSNs act as integrators for converging excitatory inputs from the neocortex and thalamus, and inhibitory inputs from local interneurons and other MSNs (Wilson, 1998; Kreitzer and Malenka, 2008). Although local GABAergic signaling strongly influences the activity of MSNs (Nisenbaum and Berger, 1992; Wilson, 2007), the role of GABAergic interneurons in regulating direct- or indirect-pathway MSNs remains poorly understood.

The majority of GABAergic control of MSNs arises through feedforward inhibition from local inhibitory interneurons (Tepper et al., 2004). Most feedforward inhibition is mediated by parvalbumin-expressing fast-spiking interneurons (FS), but some feedforward inhibition may also arise from low-threshold spiking interneurons (LTS) (Koós and Tepper, 1999). FS interneurons are well positioned to mediate feedforward inhibition in the striatum because they receive strong cortical innervation (Ramanathan et al., 2002) and respond with faster latency to cortical stimulation than MSNs (Mallet et al., 2005). In turn, FS interneurons project strongly to MSNs (Taverna et al., 2007). Decreased numbers of striatal FS interneurons are associated with Tourette syndrome (Kalanithi et al., 2005) and dystonia (Gernert et al., 2000); FS cells have also been hypothesized to amplify imbalances in striatal output during Parkinson disease (Mallet et al., 2006). Although these studies highlight the importance of GABAergic interneurons in normal striatal function, it is not known whether feedforward inhibition differentially affects MSNs in the direct or indirect pathway. In part, this reflects historic difficulties in identifying distinct striatal cell types in acute 
brain slices, particularly GABAergic interneurons which make up $<5 \%$ of all striatal neurons. In this paper, we use BAC transgenic mouse lines to identify GABAergic interneurons, direct-pathway MSNs, and indirect-pathways MSNs in single brain slices to address the role of interneuron subtypes in regulating striatal output pathways.

\section{Materials and Methods}

Electrophysiological recordings. Coronal sections (250-300 $\mu \mathrm{m}$ thickness) containing dorsal striatum were prepared from brains of 3- to 5-weekold heterozygous Lhx6-GFP BAC transgenic mice or Lhx6-GFP mice crossed to Drdla-tdTomato BAC (GENSAT project, www.gensat.org) on a FVB background. Sections were bisected to generate left and right hemisphere slices. Slices were prepared with a Leica VT1000S vibratome in carbogenated ACSF containing (in mM): $125 \mathrm{NaCl}, 26 \mathrm{NaHCO}_{3}, 2.5$ $\mathrm{KCl}, 1 \mathrm{MgCl}_{2}, 2 \mathrm{CaCl}_{2}, 1.25 \mathrm{NaH}_{2} \mathrm{PO}_{4}, 12.5$ glucose, $2 \mathrm{Mg}$-ATP, and 0.3 Na-GTP, pH 7.25 or a sucrose cutting solution (in mM): $79 \mathrm{NaCl}, 23$ $\mathrm{NaHCO}_{3}, 68$ sucrose, 12 glucose, $2.3 \mathrm{KCl}, 1.1 \mathrm{NaH}_{2} \mathrm{PO}_{4}, 6 \mathrm{MgCl}_{2}$, and 0.5 $\mathrm{CaCl}_{2}$. After recovery for $30 \mathrm{~min}-5 \mathrm{~h}$, experiments were performed on slices perfused with $\mathrm{ACSF}$, warmed to $31-33^{\circ} \mathrm{C}$. In experiments that measured excitatory transmission, $50 \mu \mathrm{m}$ picrotoxin (Sigma) was included in the perfusion saline.

Data were collected with a MultiClamp 700B amplifier (Molecular Devices) and ITC-18 A/D board (HEKA) using Igor Pro software (Wavemetrics) and custom acquisition routines (mafPC, courtesy of M. A. $\mathrm{Xu}$-Friedman). Current-clamp recordings were filtered at $10 \mathrm{kHz}$ and digitized at $40 \mathrm{kHz}$; voltage-clamp recordings were filtered at $2 \mathrm{kHz}$ and digitized at $10 \mathrm{kHz}$. Electrodes were made from borosilicate glass (pipette resistance, 2-4 M $\Omega$ ).

Action potential half-width was calculated as the width of the action potential, measured at a point half-way between threshold and AP peak. AP threshold was defined as the voltage at which the acceleration in voltage exceeded $3 \times 10^{5} \mathrm{~V} / \mathrm{s} / \mathrm{s}$. Maximum firing rate was measured as the average maximum firing rate over a $500 \mathrm{~ms}$ step that could be sustained without entering depolarization block. Input resistance was calculated in voltage clamp as the deviation from the holding current caused by a $5 \mathrm{mV}$ hyperpolarizing step. The change in current was calculated as the difference between baseline holding current and the average current during the last $10 \mathrm{~ms}$ of the $40 \mathrm{~ms}$ hyperpolarizing voltage step. Wholecell capacitance was calculated by integrating the area under the transient following a $5 \mathrm{mV}$ hyperpolarizing voltage step from the holding potential, $-70 \mathrm{mV}$. The holding current was subtracted before integrating. $R_{\text {series }}$ was calculated by fitting an exponential to the decay of the transient and using $\tau=R_{\text {series }} \times$ whole-cell capacitance. $R_{\text {series }}$ was $6.4 \pm 1.8$ $\mathrm{M} \Omega$ for dynamic clamp experiments, $5.9 \pm 2.1 \mathrm{M} \Omega$ for postsynaptic MSNs in paired recordings, and $8.1 \pm 3.8 \mathrm{M} \Omega$ for interneurons in paired recordings. Because synaptic currents were usually $<500 \mathrm{pA}, R_{\text {series }}$ compensation was not applied. The synaptic delay was measured from the peak of the action potential to the $10 \%$ rise of the IPSC.

Different internal solutions were used, depending on the experiment. (1) To measure neuronal excitability and record from presynaptic interneurons in interneuron-MSN pairs, the internal solution contained (in $\mathrm{mm}$ ): $130 \mathrm{KMeSO}_{3}, 10 \mathrm{NaCl}, 2 \mathrm{MgCl}_{2}, 0.16 \mathrm{CaCl}_{2}, 0.5$ EGTA, 10 HEPES, $2 \mathrm{Mg}$-ATP, $0.3 \mathrm{Na}$-GTP, pH 7.25 to classify GFP+ neurons as FS or PLTS based on their intrinsic firing properties (Fig. 1). (2) To measure excitatory inputs onto interneurons, the internal solution contained (in $\mathrm{mM}$ ): $120 \mathrm{CsMeSO}_{3}, 15 \mathrm{CsCl}, 8 \mathrm{NaCl}, 0.5$ EGTA, 10 HEPES, $2 \mathrm{Mg}$-ATP, 0.3 Na-GTP, 5 QX-314, pH 7.3. (3) In paired recordings, postsynaptic MSNs were patched with an internal solution containing (in mM): $120 \mathrm{CsCl}, 15$ $\mathrm{CsMeSO}_{3}, 8 \mathrm{NaCl}$, 0.5 EGTA, 10 HEPES, 2 Mg-ATP, 0.3 Na-GTP, 5 QX-314, pH 7.3. (4) To measure synaptic connections between interneurons, the internal solution contained (in $\mathrm{mM}$ ): $60 \mathrm{KCl}, 75 \mathrm{KMeSO}_{3}, 8$ NaCl, 0.5 EGTA, 10 HEPES, 2 Mg-ATP, 0.3 Na-GTP, pH 7.25.

Histology and immunohistochemistry. Tissue preparation was performed as described previously (Palop et al., 2005). Briefly, mice were perfused transcardially with phosphate buffered saline (PBS), followed by $4 \%$ paraformaldehyde in PBS. Brains were removed and drop-fixed with the same fixative at $4^{\circ} \mathrm{C}$ for $48 \mathrm{~h}$. After rising with PBS, brains were transferred to $30 \%$ sucrose in PBS and stored at $4^{\circ} \mathrm{C}$ for $24 \mathrm{~h}$ before sectioning. Thin coronal sections $(30 \mu \mathrm{m})$ were made through forebrains of 5-week old Lhx6-GFP BAC transgenic mice. Experiments were done on a series of sections, consisting of 5-6 bisected sections, $300 \mu \mathrm{m}$ apart which spanned the rostral-caudal extent of the striatum. For quantification, cell counts were performed on the most rostral 1-2 sections per animal and were restricted to the dorsal $2 / 3$ of the striatum (Fig. $1 A$ ). The tissue was blocked with $10 \%$ normal donkey serum and permeabilized with $0.5 \%$ Triton X-100. Primary antibody incubations were performed at $4^{\circ} \mathrm{C}$ for $48 \mathrm{~h}$, using either rabbit anti-PV (1:5000, Swant), rabbit antiNPY (1:2000, Immunostar), rat anti-SOM (1:1000, Millipore Bioscience Research Reagents), or rabbit anti-CR (1:3000, Swant). Primary antibodies were detected with Alexa Fluor 594-conjugated goat antirabbit (1:500, Vector Laboratories), Alexa Fluor 594-conjugated goat anti-rat (1:500, Vector Laboratories), or Alexa Fluor 633-conjugated goat anti-rabbit (1:500 Vector Laboratories), incubated for $2 \mathrm{~h}$. at room temperature.

For cell fills of PLTS interneurons, $0.3 \%$ biocytin was dissolved in $\mathrm{KMeSO}_{3}$ internal solution and recordings from PLTS interneurons were obtained. After electrophysiological verification of PLTS properties, cells were filled for 20-30 min, and the pipette was subsequently withdrawn from the neuron. The slice was fixed in $4 \%$ PFA overnight at $4^{\circ} \mathrm{C}$. The following day, slices were washed in PBS, permeabilized in 5\% PBSTriton X-100 for 30 min then $0.5 \%$ PBS-Triton X-100 overnight, and developed using the $\mathrm{AB}$ Complex $(\mathrm{ABC}), 3,3^{\prime}$-diaminobenzidine (DAB) kit from Vector Laboratories.

Dynamic clamp. See supplemental methods for detailed description. Briefly, dynamic clamp was performed using mafPC software routines in Igor Pro. The kinetics and $G_{\max }$ of excitatory and inhibitory conductances were modeled after experimentally measured values $\left(\tau_{\mathrm{e}}=5.1 \mathrm{~ms}\right.$; $\left.\tau_{\mathrm{i}}=6.7 \mathrm{~ms}\right)$. Excitatory $G_{\text {reversal }}$, and inhibitory $G_{\text {reversal }}$ were set at $0 \mathrm{mV}$ and $-65 \mathrm{mV}$, respectively. To mimic high-frequency inputs that MSNs experience during an 'up-state', we injected excitatory conductances at random frequencies ranging from 300 to $600 \mathrm{~Hz}$. Lower frequencies did not drive spiking reliably in MSNs and higher frequencies were not well tolerated. The same stimuli were presented in multiple neurons to measure cell-to-cell variability rather than pattern-to-pattern variability. A total of three different stimuli at each frequency were used.

To minimize the variability that would arise from adding random inhibitory conductances onto random excitatory conductances, we kept the interval of inhibitory inputs constant. Inhibitory frequencies were based on the assumption that: (1) any given FS interneuron operates across a dynamic range of 10-100 Hz (Berke et al., 2004; Berke, 2008) (2) MSNs receive converging inputs from 4-27 FS interneurons (Koós and Tepper, 1999) and (3) FS interneurons do not all fire synchronously (Berke, 2008). To mimic inputs from 4 inhibitory interneurons, each firing at $50 \mathrm{~Hz}$, the frequency of inhibitory conductances was set to $4 \times$ $50 \mathrm{~Hz}=200 \mathrm{~Hz}$. Although this assumes that all 4 neurons are firing perfectly out-of-phase with each other, we are using a lower-bound estimate for inhibitory convergence, and the probability of unsynchronized inputs would only increase with more FS neurons.

\section{Results}

Lhx6-GFP mouse line labels striatal GABAergic interneurons The LIM homeobox protein 6 (Lhx6) is expressed by cells in the medial ganglionic eminence that develop into parvalbumin (PV)-, somatostatin (SOM)-, calretinin (CR)-, and neuropeptide $\mathrm{Y}(\mathrm{NPY})$-expressing interneurons in the neocortex (Cobos et al., 2006) and striatum (Marin et al., 2000). To determine whether the Lhx6-GFP BAC transgenic mouse line can be used to efficiently target striatal GABAergic interneurons in acute brain slices, we recorded from GFP + and GFP- neurons in the striatum of 3- to 5-week-old Lhx6-GFP transgenic mice. As shown in Figure $1 A$, neurons labeled with GFP were scattered throughout the striatum of Lhx6-GFP mice and accounted for $2.6 \%$ of all striatal neurons, labeled with the neuronal marker NeuN. 
A

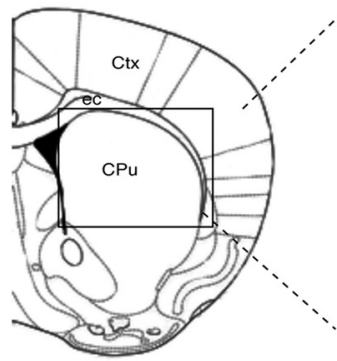

C

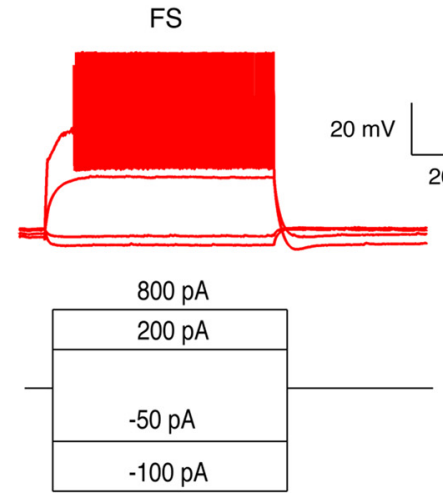

D
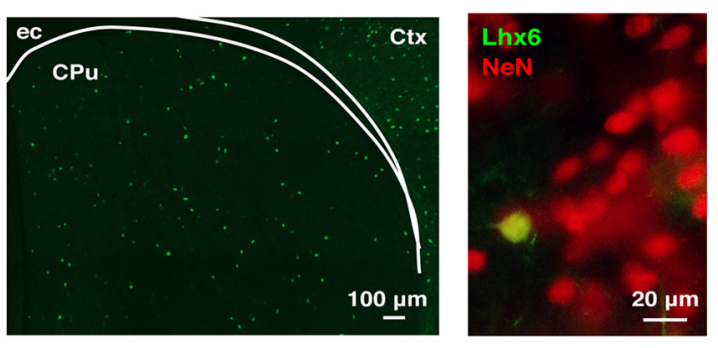

B

Percentage of GFP+ neurons

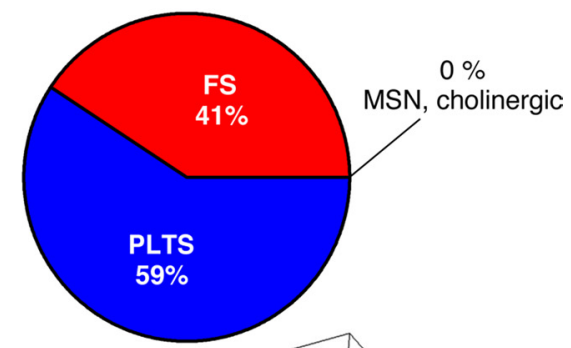

PLTS

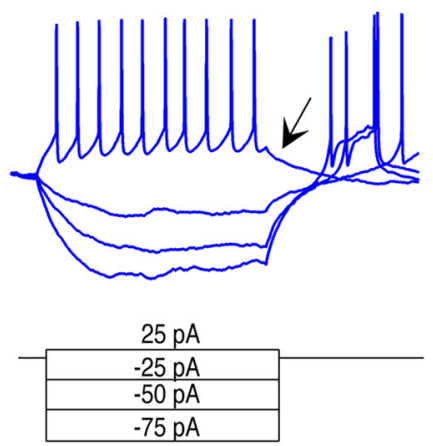

E

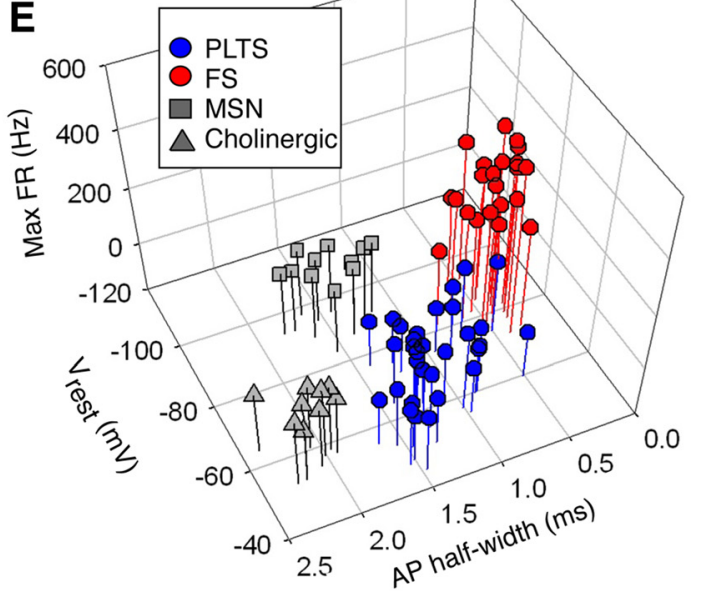

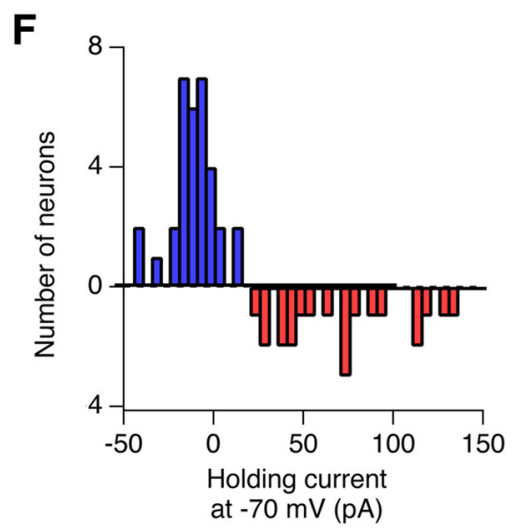

G

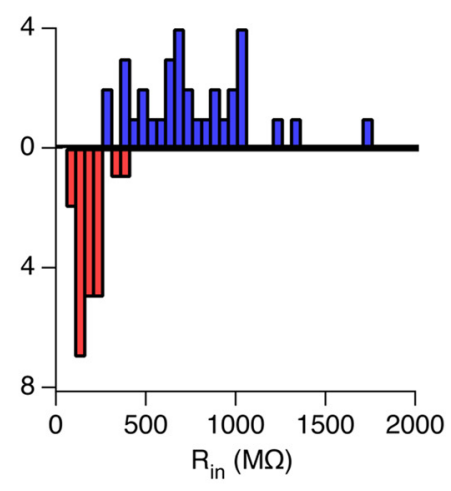

H

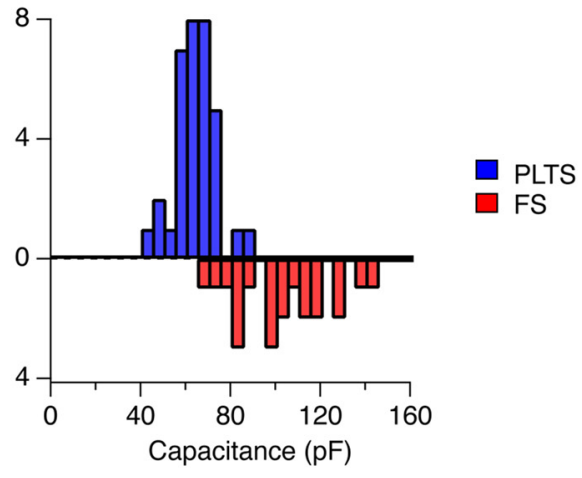

Figure 1. Striatal GABAergic interneurons can be targeted with GFP fluorescence in Lhx6-GFP BAC transgenic mice. $\boldsymbol{A}$, Middle: GFP expression in the dorsal striatum of a 1-month-old Lhx6 mouse. Left: The region of striatum shown is indicated with a box on the schematic. Ctx, Cortex; CPu, caudate-putamen; ec, external capsule. Right: An image at 20X magnification showing that GFP expression is restricted to a small percentage of striatal neurons labeled with NeuN. B, Percentage of GFP + neurons recorded in Lhx6 mice that had electrophysiological properties of distinct striatal cell types. All GFP + neurons had firing properties of either FS or PLTS GABAergic interneurons but never of MSNs or cholinergic interneurons. $\boldsymbol{C}, \boldsymbol{D}$, Current-clamp recordings, showing the response of a typical FS interneuron $(\boldsymbol{C})$ and PLTS interneuron $(\boldsymbol{D})$ to $500 \mathrm{~ms}$ current steps. Arrow in $\boldsymbol{D}$ indicates a plateau potential that persists after the offset of depolarizing current, observed in most PLTS interneurons. $\boldsymbol{E}$, Striatal cell types form distinct clusters on a 3-D plot of firing properties. MSNs, cholinergic interneurons, FS interneurons, and PLTS interneurons could be distinguished by their maximum firing rates (maxFR), resting membrane potentials $\left(V_{\text {rest }}\right)$, and action potential half-widths. $\boldsymbol{F}$ - $\boldsymbol{H}$, Histograms showing distinct passive membrane properties of FS (red) and PLTS interneurons (blue), measured in voltage-clamp at a holding potential of $-70 \mathrm{mV}$. The cell types had significantly different holding currents $(\boldsymbol{F})$, input resistance $(\boldsymbol{G})$, and whole-cell capacitance $(\boldsymbol{H})$.

Differences in the electrophysiological properties of striatal cell types are well documented (Kreitzer, 2009), enabling our identification of MSNs, FS, PLTS, and cholinergic interneurons based on a combination of passive and active membrane properties (Table 1). Figure $1 E$ demonstrates the use of three parameters (maximum firing rate, $V_{\text {rest }}$, and action potential half-width) to distinguish all four classes of striatal neurons. All of the 55 GFP+ neurons targeted in the Lhx6-GFP line had electrophysiological properties of either PLTS (59\%) or FS (41\%) interneurons (Fig. $1 B-D)$, but not MSNs or cholinergic interneurons. In current clamp, FS interneurons can be distinguished from PLTS interneurons by their narrower action potentials $(p<0.0001)$, higher maximum firing rates $(p<0.0001)$, and lower resting membrane potentials $(p<0.0001)$ (Fig. $1 C-E)$. In voltage clamp at $V_{\text {hold }}=-70 \mathrm{mV}$, FS interneurons have more positive holding currents $(p<0.0001)$, lower input resistances $(p<0.0001)$, and higher cell capacitances $(p<0.0001)$ compared with PLTS interneurons (Fig. $1 F-H$ ). These data demonstrate that striatal GABAergic interneurons are selectively labeled in the Lhx6-GFP $\mathrm{BAC}$ transgenic mouse line, which therefore represents a new tool for targeting these interneurons for electrophysiological study in acute brain slices.

Table 2 shows the enhanced success rate for targeting GABAergic interneurons in the striatum using the Lhx6-GFP line 
Table 1. Intrinsic properties of striatal neurons: summary of cell parameters collected from GFP-positive PLTS and FS interneurons as well as GFP-negative MSNs and cholinergic interneurons

\begin{tabular}{|c|c|c|c|c|}
\hline & PLTS & FS & MSN & Cholinergic \\
\hline & 32 & 22 & 11 & 9 \\
\hline AP half-width (ms)* & $1.3 \pm 0.3$ & $0.5 \pm 0.1$ & $1.5 \pm 0.2$ & $2.1 \pm 0.2$ \\
\hline $\operatorname{MaxFR}(\mathrm{Hz})^{*}$ & $29 \pm$ & $287 \pm 88$ & $69 \pm$ & $60 \pm 18$ \\
\hline$V_{\text {rest }}(\mathrm{mV})^{*}$ & $-61 \pm 10$ & $-82 \pm 5$ & $-92 \pm 4$ & $-57 \pm 4$ \\
\hline Plateau potential $(\mathrm{mV})^{*}$ & $6.4 \pm 5.2$ & $-1.7 \pm 2.4$ & $0.7 \pm 0.7$ & $-6.1 \pm 6.7$ \\
\hline Holding current at $-70 \mathrm{mV}(\mathrm{pA})^{\dagger}$ & $-14 \pm 15$ & $67 \pm 37$ & $207 \pm 25$ & $-99 \pm 60$ \\
\hline Input resistance $(M \Omega)^{\dagger}$ & $830 \pm 525$ & $198 \pm 126$ & $142 \pm 60$ & $229 \pm 69$ \\
\hline Capacitance $(\mathrm{pF})^{\dagger}$ & $65 \pm 8$ & $98 \pm 24$ & $118 \pm 22$ & $98 \pm 12$ \\
\hline
\end{tabular}

Each cell type is easily distinguished using various combinations of these parameters. *Parameters measured in current clamp; ${ }^{\dagger}$ parameters measured in voltage clamp.

Table 2. Probabilities of finding striatal cell types when targeting interneurons in acute brain slices

\begin{tabular}{lccc}
\hline & Lhx6-EGFP & D1-D2-XFP & Wild type \\
\hline FS & $21 / 55$ & $22 / 60$ & $8 / 83$ \\
PLTS & $34 / 55$ & $23 / 60$ & $8 / 83$ \\
Chol & $0 / 55$ & $10 / 60$ & $20 / 83$ \\
MSN & $0 / 55$ & $5 / 60$ & $47 / 83$ \\
\hline
\end{tabular}

The Lhx6-EGFP line enables highly efficient targeting of striatal interneurons. All fluorescent neurons targeted in Lhx6-EGFP mice were GABAergic interneurons. In contrast, targeting nonfluorescent neurons in D1-D2-XFP mice yielded GABAergic interneurons $75 \%$ of the time. Note that $<10 \%$ of nonfluorescent neurons in D1-D2-XFP mice were MSNs, suggesting highly efficient labeling of D1- and D2-MSNs by D1-Imt and D2-GFP fluorescence, respectively. The last column shows the low success rate for targeting GABAergic interneurons using traditional methods in wild-type mice.

relative to other approaches, including the traditional approach of visual identification in wild-type mice, or crossing a Drd2-GFP BAC transgenic mouse line with a Drdla-tdTomato BAC transgenic line (Shuen et al., 2008) (D1-D2-XFP) and recording from nonfluorescent neurons. All fluorescent neurons targeted in Lhx6-GFP mice are GABAergic interneurons, enabling rapid and reliable identification of this sparse neuronal population in acute brain slices, significantly advancing our ability to study the roles of these interneurons in shaping striatal microcircuits.

\section{Fluorescently labeled neurons in Lhx6-GFP transgenic mice express PV or NPY/SOM}

FS GABAergic interneurons in the striatum express parvalbumin (PV) while PLTS interneurons express neuropeptide Y (NPY), somatostatin (SOM), and nitric oxide synthase (NOS) (Kawaguchi et al., 1995). To verify that these interneuron markers are expressed in GFP + neurons in the Lhx6 line, we performed immunostains on a series of $30 \mu \mathrm{m}$ sections collected from three 1-month-old Lhx6-GFP BAC transgenic mice. From each mouse, one series of sections was stained with antibodies against PV (Fig. 2A) and another with antibodies against NPY and SOM (Fig. $2 B$ ). The number of neurons expressing each marker was pooled across sections.

In sections labeled with antibodies against NPY and SOM, $61 \%(257 / 420)$ of GFP + neurons expressed one or both markers, which showed $>90 \%$ overlap in the striatum. In sections labeled with antibodies against PV, 34\% (136/403) of GFP+ neurons were labeled. As shown in Figure $2 C$, combining the cell counts from these sections suggests that $95 \%$ of GFP + neurons in the Lhx6 mouse line express either NPY/SOM or PV. The ratio of NPY/SOM labeled neurons relative to that of PV labeled neurons was very similar to the ratio of PLTS to FS interneurons measured electrophysiologically in Figure 1, further validating the Lhx6GFP line for targeting GABAergic interneurons in the striatum.

To determine the identity of the remaining $5 \%$ of unlabeled GFP + neurons, we performed an additional immunostain for the interneuron marker calretinin (CR), which labels a distinct class of interneurons in the striatum (Figueredo-Cardenas et al., 1996). In contrast to primates and rats, CR staining was sparse in the striatum of mice (supplemental Fig. 1, available at www. jneurosci.org as supplemental material). Although we observed robust CR staining in the neocortex, a total of only $47 \mathrm{CR}+$ neurons were counted across 3 striatal sections, in contrast to 626 $\mathrm{GFP}+$ neurons. Only 17 of the CR + neurons expressed GFP, therefore $3 \%$ of previously unlabeled GFP + neurons likely express CR. Together, $\sim 98 \%$ of GFP + neurons in Lhx6-GFP mice were labeled with antibodies against PV, NPY/SOM, or CR.

To estimate the fraction of all PV or NPY/SOM neurons that express GFP in Lhx6-GFP mice, we counted the total number of neurons expressing these markers in our sections. A high percentage of NPY/SOM + neurons $(81 \%, 257 / 318)$ were labeled by GFP while only approximately half of $\mathrm{PV}+$ neurons were labeled $(47 \%, 136 / 289)$. Although the population of PV + interneurons is not fully represented in the Lhx6-GFP line, a comparison of the firing properties of FS interneurons recorded in Lhx6-GFP mice relative to those targeted in other mouse lines yielded nearly overlapping firing properties with only a small difference in action potential half-width (supplemental Fig. 2, available at www. jneurosci.org as supplemental material). This indicates that GFP expression does not label an electrophysiologically distinct subpopulation of FS interneurons in Lhx6-GFP mice.

\section{Excitatory synapses onto FS and PLTS interneurons have distinct properties}

To measure excitatory inputs onto FS and PLTS interneurons, $\mathrm{GFP}+$ neurons were patched in whole-cell voltage-clamp configuration $\left(V_{\text {hold }}=-70 \mathrm{mV}\right)$ in the presence of picrotoxin $(50 \mu \mathrm{M})$, to block fast GABAergic signaling. In some of the experiments, a K-based internal solution was used to distinguish FS and PLTS interneurons, using physiological firing properties as described above (FS: $n=19$, PLTS $n=9$ ). In a subset of experiments requiring depolarization of neurons, a Cs-based internal solution containing QX-314 was used to block action potentials and improve voltage-clamp (FS: $n=9$, PLTS $n=24$ ). In these latter experiments, FS and PLTS interneurons were readily identified by their characteristic input resistance, capacitance, and holding potential (at $-70 \mathrm{mV}$ ) measured immediately upon break-in. The choice of internal solution did not significantly affect the amplitude or kinetics of the EPSCs (K-based vs Cs-based internal: FS amp, $p=0.24$; PLTS amp, $p=0.19$; FS decay, $p=0.53$; PLTS decay $p=0.47$ ), so data were pooled.

Once an interneuron was identified as FS or PLTS, a glass stimulating electrode containing saline was placed in the striatum. Small currents, $0.3 \mathrm{~ms}$ in duration, were passed across the stimulating electrode to evoke EPSCs from excitatory striatal afferents. In FS interneurons, fast and large EPSCs $(255 \pm 119 \mathrm{pA}$, $n=20)$ were evoked at small stimulation amplitudes $(5.5 \pm 2.7$ $\mu \mathrm{A}$ ) and increased in size as a function of stimulus intensity (Fig. $3 A, C)$. In contrast, higher stimulus intensities were required to evoke EPSCs in PLTS interneurons. At an average stimulus intensity of $9.7 \pm 3.0 \mu \mathrm{A}$, EPSCs in PLTS interneurons were $86.2 \pm$ $60 \mathrm{pA}, n=31$ and rarely exceeded $200 \mathrm{pA}$, even at stimulus intensities between 20 and $50 \mu \mathrm{A}$ (Fig. $3 B, C$ ).

To investigate the probability of release at excitatory synapses onto interneurons, the paired-pulse ratio (PPR) was calculated. Two pulses were given with a variable interstimulus interval (25-500 ms), and the amplitude ratio of $\mathrm{EPSC}_{2} / \mathrm{EPSC}_{1}$ was measured (bottom, Fig. $3 A, B)$. FS interneurons demonstrated synaptic facilitation at short interstimulus intervals, in contrast to PLTS interneurons, 
A

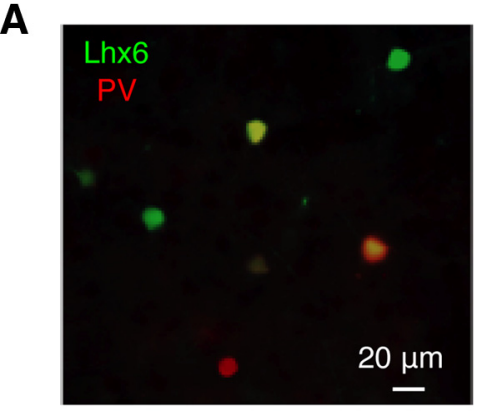

B

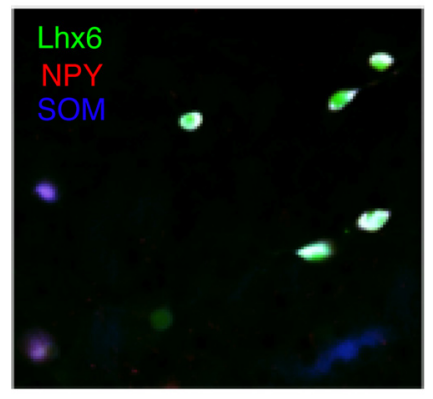

C

Percentage of GFP+ neurons

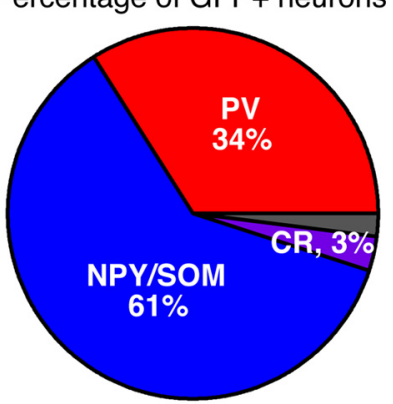

D

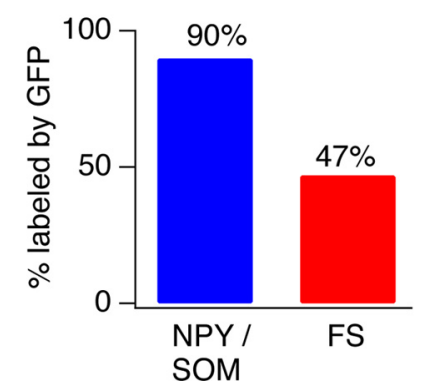

Figure 2. GFP+ neurons in the Lhx6-GFP BAC transgenic line express markers of striatal interneurons. $A$, Results of immunostaining in dorsal striatum of an Lhx6-GFP BAC transgenic mouse, showing colabeling of GFP + neurons with PV. Double labeled neurons are yellow. The populations were partially overlapping; $34 \%$ of GFP + neurons were labeled with an antibody for PV and $50 \%$ of PV + neurons expressed GFP. $\boldsymbol{B}$, Results of immunostaining in dorsal striatum of an Lhx6-GFP BAC transgenic mouse, showing colabeling of GFP + neurons with NPY and SOM. Triple labeled neurons are white. NPY and SOM expression was highly overlapping; $93 \%$ of NPY + neurons were also SOM + and $91 \%$ of SOM + neurons were also NPY + . C, Pie graph summarizing the percentage of GFP + neurons that were labeled with PV, NPY/SOM, or CR. Percentages were calculated by counting all GFP+ neurons from 1 to 2 hemispheres of dorsal striatum from 2 to 3 Lhx6 transgenic mice. Ninety-eight percent of all GFP + neurons were positive for one of the 3 interneuron markers. $D$, Bar graph showing the percentage of all PV + and NPY/SOM + neurons counted that expressed GFP.

which showed modest paired-pulse depression at all intervals tested. These results are consistent with a model in which FS interneurons receive a large number of synaptic inputs with a relatively lower release probability, and PLTS interneurons receive just a few synaptic inputs with a relatively higher release probability.

The postsynaptic features of excitatory synapses were investigated by determining the voltage dependence of EPSCs, as well as the AMPA and NMDA receptor contributions to postsynaptic potentials. EPSCs exhibited more rapid decay kinetics in FS versus PLTS interneurons $(1.5 \pm 0.1 \mathrm{~ms}, n=28$ vs $3.50 \pm 0.30 \mathrm{~ms}$, $n=33, p<0.0001$ ) (Fig. $3 D$ ). In FS interneurons, large EPSCs were evoked at hyperpolarized holding potentials, whereas at depolarized potentials, virtually no outward current was detected (Fig. 3E) indicating the presence of GluR2-lacking AMPA receptors and the absence of NMDA receptors. Across all FS interneurons, the voltage dependence of the peak EPSC amplitude showed marked rectification at depolarized potentials. In contrast, EPSCs in PLTS interneurons were composed of a relatively rapid AMPA-mediated component and a slower NMDAmediated component, which was blocked by bath application of D-APV $(50 \mu \mathrm{M})$ (Fig. $3 E$ ). To calculate the ratio of AMPA- to NMDA-receptor-mediated components of the EPSC in PLTS interneurons, the AMPA-mediated conductance, measured at -70 $\mathrm{mV}$ was compared with the NMDA-mediated conductance, measured at $+40 \mathrm{mV}$. The average AMPA/NMDA ratio was $1.4 \pm 1.0$ $(n=6)$ in PLTS interneurons (Fig. $3 F)$, indicating a relatively large NMDA component. The voltage dependence of the residual AMPAmediated component of the EPSC in APV showed only mild rectification, indicating the presence of GluR2-containing AMPA receptors (Fig. 3E). The presence of calcium-permeable GluR2-lacking AMPA receptors and undetectable NMDA receptors at FS inputs, compared with the presence of calcium-impermeable GluR2containing AMPA receptors and NMDA receptors at PLTS inputs, suggests that distinct forms of long-term synaptic plasticity may be expressed at these synapses.

\section{PLTS interneurons are sparsely connected to other striatal neurons}

To measure inhibitory inputs from PLTS interneurons onto MSNs, paired wholecell recordings were established between presynaptic PLTS interneurons and postsynaptic MSNs within a $250 \mu \mathrm{m}$ radius (average distance sampled $=153 \pm 80 \mu \mathrm{m}$ ). A high-chloride Cs-based internal solution was used to record from postsynaptic MSNs in the voltage-clamp configuration ( $V_{\text {hold }}=-70 \mathrm{mV}$ ) to better resolve GABAergic synaptic transmission. Presynaptic PLTS interneurons were targeted using GFP fluorescence in Lhx6-GFP mice and identified electrophysiologically, using a combination of voltage-clamp and current-clamp parameters (Fig. 1, Table 1). In recordings from 60 pairs of neurons, functional connections were only observed twice, consistent with the sparse axonal morphology of PLTS interneurons (Kawaguchi, 1993). PLTS synapses recorded in MSNs were very weak and displayed asynchronous release (11 pA and $28 \mathrm{pA}$ ) (Fig. $4 A-E$ ). Because pairs of action potentials can be insufficient to reveal functional connections at some synapses (Földy et al., 2007), we conducted additional tests for connectivity in 16 pairs using trains of 10 action potentials, at frequencies ranging from 10 to $100 \mathrm{~Hz}$ (Fig. $4 B, D)$. Higher levels of presynaptic activity did not reveal any additional functional connections.

Given the abundance of PLTS interneurons in the striatum, it was surprising that their inhibitory projections onto MSNs were weak and sparse. We hypothesized that perhaps PLTS interneurons more robustly inhibit other classes of striatal neurons, such as other PLTS, FS, or cholinergic interneurons. To test this hypothesis, we recorded from pairs of interneurons using a $\mathrm{KMeSO}_{3}$ internal solution with high $\left[\mathrm{Cl}^{-}\right]_{\mathrm{i}}$ to set $\mathrm{E}_{\mathrm{Cl}}=-20 \mathrm{mV}$. This enables better detection of GABAergic synaptic transmission and allowed bidirectional tests for connectivity. However, PLTS connectivity was similarly sparse onto all classes of striatal neurons (Fig. $4 E$ ). No functional connections were observed between PLTS-PLTS pairs (0/26), PLTS-FS pairs (0/8), or PLTScholinergic pairs $(0 / 10)$. To ensure that the axons of PLTS interneurons were preserved in our slices, the morphology of 7 biocytin-filled PLTS interneurons was examined (Fig. $4 F$ ). PLTS axons were well preserved in our coronal sections (average axon length: $144 \pm 51 \mu \mathrm{m}, n=7$ ), indicating that the angle of the slice preparation is unlikely to account for the low connectivity of PLTS interneurons. Together, these data indicate that PLTS interneurons are unlikely to provide a major source of feedforward inhibition in the striatum. 
FS interneurons mediate feedforward inhibition in the striatum

In contrast to PLTS interneurons, FS interneurons were robustly connected to MSNs within a $250 \mu \mathrm{m}$ radius (average distance sampled $=106 \pm 25 \mu \mathrm{m}$ ) (Fig. 5A-C). A high-chloride $\mathrm{CsMeSO}_{3}$ internal solution was used to record from postsynaptic MSNs in the voltage-clamp configuration $\left(V_{\text {hold }}=\right.$ $-70 \mathrm{mV}$ ). Experiments were initiated by establishing whole-cell recordings from FS interneurons (identified using a combination of voltage-clamp and current-clamp parameters; Fig. 1, Table 1). Once a recording was successfully obtained, postsynaptic MSNs were targeted for recording. Typically 1-8 (average 2.4) MSNs were sampled per interneuron.

Synaptic connections were found between $45 \%$ of FS-MSN pairs (75/167). The amplitudes of unitary IPSCs (uIPSCs) measured in postsynaptic MSNs were bimodally distributed, with one peak at 65.3 $\mathrm{pA}$ and the other at $1185 \mathrm{pA}$, corresponding to peak conductances of $0.9 \mathrm{nS}$ and $16.9 \mathrm{nS}$, respectively (Fig. $5 B$ ). This bimodal distribution is unlikely to represent distinct classes of FS interneurons because the same presynaptic FS interneuron could evoke either large or small uIPSCs depending on the postsynaptic partner. In one example, the same FS interneuron evoked a uIPSC of $1491 \mathrm{pA}$ in one MSN, $1109 \mathrm{pA}$ in another, and $304 \mathrm{pA}$ in a third MSN. Unitary IPSCs exhibited rapid rise $(1.2 \pm 1.2 \mathrm{~ms})$ and decay kinetics $(8.0 \pm$ $3.2 \mathrm{~ms}, n=68)$, low failure rates $(<1 \%)$, synaptic delays of $0.88 \pm 0.23 \mathrm{~ms}$ and paired-pulse ratios $<1(0.67 \pm 0.22 \mathrm{~ms}$, $n=46$ ), indicative of a high probability of transmitter release.

In addition to GABAergic projections onto MSNs, FS interneurons also formed GABAergic synapses onto other FS interneurons, but not PLTS or cholinergic interneurons (Fig. 5D,E). In these experiments, interneurons were recorded with $\mathrm{KMeSO}_{3}$ internal solution containing a high $\left[\mathrm{Cl}^{-}\right]_{\mathrm{i}}$ to set $\mathrm{E}_{\mathrm{Cl}}=-20 \mathrm{mV}$. This enabled identification of FS interneurons based on voltage-clamp and current-clamp parameters while increasing the driving force of $\mathrm{Cl}^{-}$to better detect GABAergic synaptic transmission. The probability of finding a synaptic connection between pairs of FS interneurons was 58\% (7/12), and 3 of 4 connected pairs were reciprocally connected. The conductance of FS-FS synapses was significantly lower than that of FS-MSN synapses $(1.1 \pm 1.5 \mathrm{nS}, n=6$ vs $7.8 \pm 11.2 \mathrm{nS}, n=69 ; p=0.009)$ (Fig. $5 F$ ) but the two types of synapses had similar decay kinetics $(5.7 \pm 2.7 \mathrm{~ms}$ vs $8.0 \pm 3.2 \mathrm{~ms} ; p=0.1)$ and paired-pulse ratios $(0.69 \pm 0.29$ vs $0.68 \pm 0.22 ; p=0.83$ ) (Fig. $5 G, H)$.

\section{Short-term dynamics of feedforward inhibition enables} effective inhibition across a broad dynamic range

Recordings from the striatum of awake behaving mice reveal that FS interneurons express average firing rates of $10-15 \mathrm{~Hz}$ and
C

E

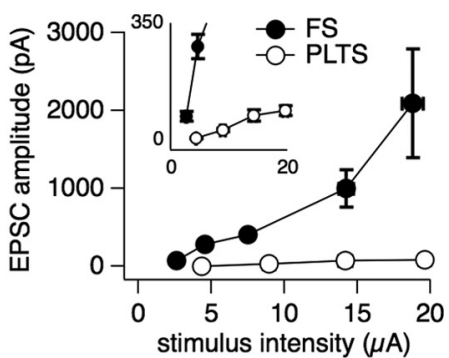

$\mathbf{F}$

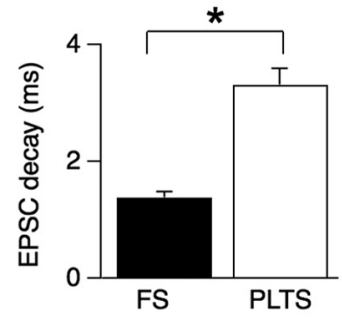

I
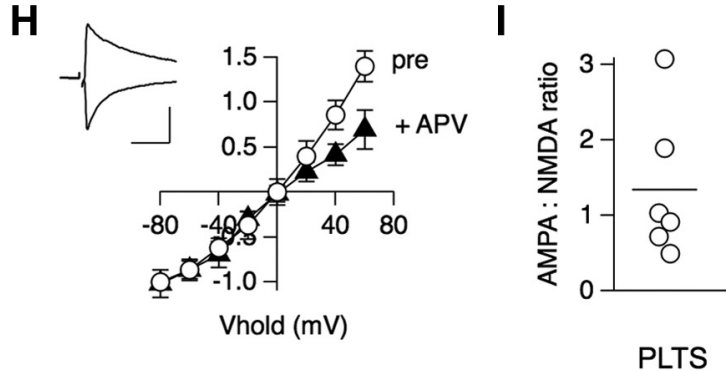

PLTS

Figure 3. Synaptic properties of excitatory afferents onto GABAergic interneurons. $A$, EPSCs elicited by intrastriatal stimulation $(5 \mu \mathrm{A}, 0.3 \mathrm{~ms})$ in an FS interneuron. $\boldsymbol{B}$, Paired-pulse ratio (PPR) as a function of the interstimulus interval for FS interneurons $(n=$ , EPSC elicited by intrastriatal stimulation $(5 \mu \mathrm{A}, 0.3 \mathrm{~ms})$ in a PLTS interneuron. $\boldsymbol{D}$, Paired-pulse ratio (PPR) as a function of were significantly larger $(p<0.0001)$ in FS $(n=24)$ compared with PLTS $(n=11)$ interneurons and increased linearly with ( 33), $p<0.0001$. *Statistical significance $(p<0.05) . \mathbf{G}, \boldsymbol{H}$, Voltage dependence of EPSCs in FS $(n=16)(\boldsymbol{G})$ and PLTS $(n=$ D-APV $(50 \mu \mathrm{M})$. Note only mild rectification observed for the AMPA-modict show typical EPSCs at $-70 \mathrm{mV}$ and $+40 \mathrm{mV}$ for each cell type. Scale bar, $200 \mathrm{pA}, 100 \mathrm{~ms}$. Error bars are SEM. I, AMPA to NMDA ratio of EPSCS in PLTS neurons. The average ratio is indicated with a line $(1.4 \pm 1.0)$.

modulate their firing rates up to $60-80 \mathrm{~Hz}$ during behavioral tasks (Berke et al., 2004; Berke, 2008). To understand how feedforward inhibition from FS interneurons affects the firing rates of MSNs (and therefore output of the striatum), it is necessary to consider how synaptic efficacy at FS synapses changes as a function of neuronal firing rate.

To measure how short-term, activity-dependent plasticity affects synaptic efficacy at FS-MSN synapses, action potentials were elicited in presynaptic FS interneurons at frequencies of 10, 20, 50 , or $100 \mathrm{~Hz}$. Short-term plasticity was measured by recording changes in the amplitude of uIPSCs in synaptically connected MSNs $\left(V_{\text {hold }}=-70 \mathrm{mV}\right)$. Trains of action potentials were delivered every $20 \mathrm{~s}$ to allow synapses time to return to baseline levels and no persistent changes in UIPSC were observed. Figure $6 \mathrm{~A}$ shows changes in UIPSCs in a postsynaptic MSN during a train of 10 action potentials evoked at different frequencies in a presynaptic FS interneuron. Due to the high $\left[\mathrm{Cl}^{-}\right]_{\mathrm{i}}$ internal used to record from MSNs, uIPSCs are inward. To calculate the amplitude of IPSCs during a train, the peak amplitude of each uIPSC was subtracted by a 'baseline value', calculated using an exponen- 

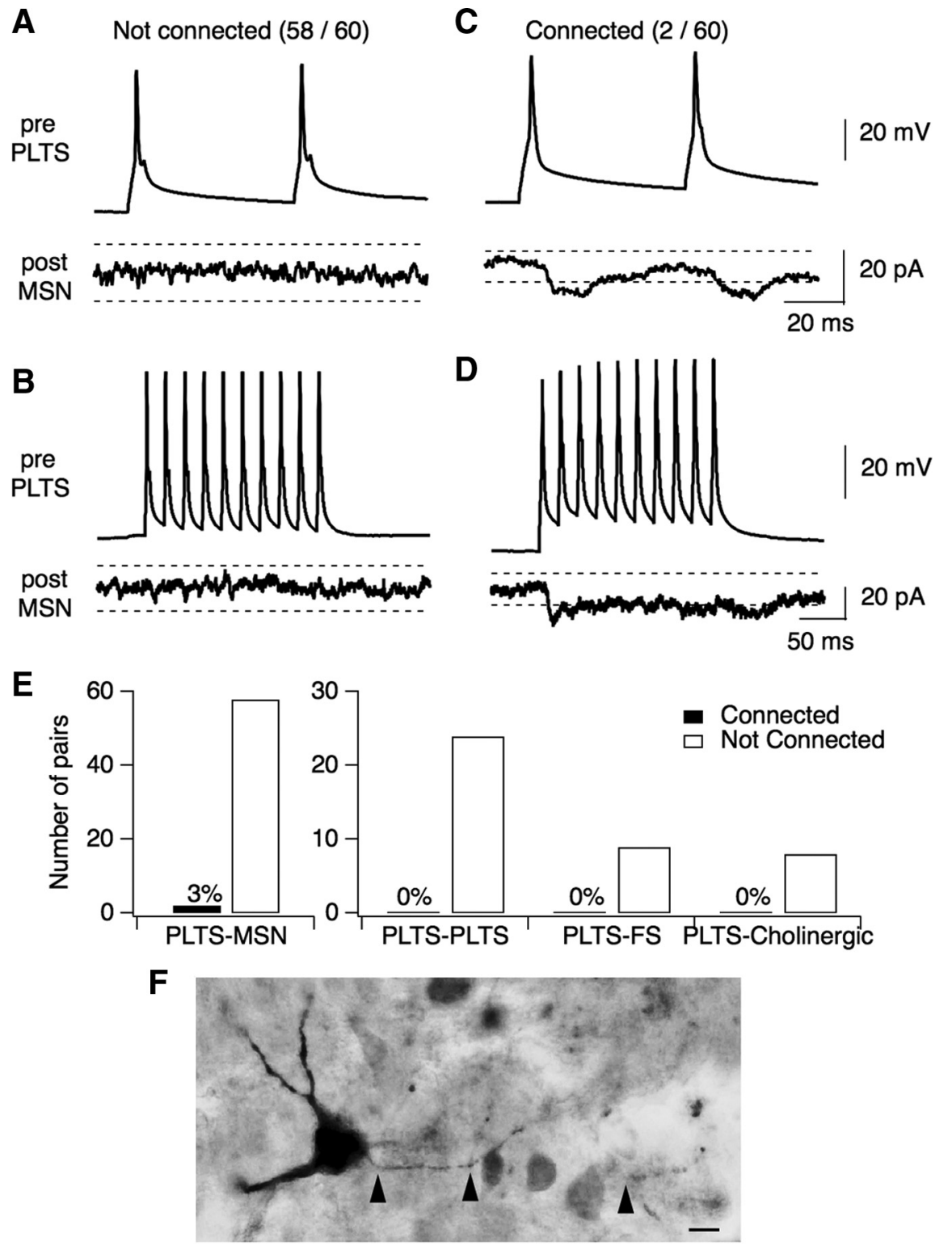

Figure 4. Local connectivity of PLTS interneurons. A, Paired recording in which no functional connection was found between a presynaptic PLTS interneuron and a postsynaptic MSN (observed for $58 / 60$ pairs). Throughout, dashed lines represent the detection threshold for synaptic events, \pm 5 SDs from the baseline noise which was typically 2-3pA. $\boldsymbol{B}$, The same pair as in $\boldsymbol{A}$, but the presynaptic cell was driven with a $50 \mathrm{~Hz}$ train of 10 action potentials. This stronger stimulus did not unmask a functional connection. $\boldsymbol{C}, \boldsymbol{D}$, Paired recording between a presynaptic PLTS interneuron and a postsynaptic MSN where a small functional connection was observed with a pair of action potentials $(\boldsymbol{C})$ and a $50 \mathrm{~Hz}$ train of 10 action potentials $(\boldsymbol{D})$. This is 1 of only 2 functional connections observed in 60 attempts. $E$, Summary of connection probabilities between PLTS interneurons and other cell types in the striatum. The number of connections found and the total number of attempts is listed for each cell type. Only 2/60 PLTS-MSN pairs were functionally connected and no connections were found between PLTS-PLTS pairs (0/26), PLTS-FS pairs (0/9), or PLTScholinergic pairs (0/8). All pairs were within a $250 \mu \mathrm{m}$ radius. $\boldsymbol{F}$, Biocytin-filled PLTS interneuron. Left arrowhead labels the axon initial segment; middle arrowhead labels the first branch point ( $\sim 60 \mu \mathrm{m}$ away from the soma); right arrowhead labels a second branch point ( $\sim 130 \mu \mathrm{m}$ away from the soma). The axon could be followed for $\sim 160 \mu \mathrm{m}$.

tial fit to the decay of the preceding IPSC. This method controls for summation of charge during a train and measures only changes in synaptic transmission.

As shown in Figure 6B, FS synapses showed marked depression at all frequencies tested: $0.34 \pm 0.16$ at $10 \mathrm{~Hz}, 0.29 \pm 0.15$ at $20 \mathrm{~Hz}, 0.23 \pm 0.13$ at $50 \mathrm{~Hz}$, and $0.17 \pm 0.08$ at $100 \mathrm{~Hz}, n=24$, $p<0.0001$ at all frequencies. Although depression changed somewhat with frequency, increasing by $11 \%$ between 10 and 50 $\mathrm{Hz}$, this change was relatively small compared with synaptic depression at synapses with canonical frequency-dependent depression (Abbott et al., 1997; Dittman and Regehr, 1998; Markram et al., 1998). This type of frequency-independent depression is a common feature of FS interneurons in other brain regions (Galarreta and Hestrin, 1998; Varela et al., 1999; Gabernet et al., 2005).

To test how the short-term dynamics of FS synapses shape the input-output functions of MSNs over rapid time scales, we used dynamic clamp. The input-output functions of MSNs were measured by calculating the average neuronal firing rates evoked by $2 \mathrm{~s}$ of simulated EPSCs (sEPSCs) at frequencies between 300 and $600 \mathrm{~Hz}$ (Fig. $6 C, D)$. This frequency range is similar to that required to drive up-state transitions in cultured MSNs (Blackwell et al., 2003). In the absence of inhibition, the firing rates of MSNs increased sublinearly as a function of input frequency (Fig. 6G).

To measure how the dynamics of feedforward inhibition affect the input-output transformations of MSNs, sEPSCs were repeated in conjunction with sIPSCs displaying either frequency-dependent or frequency-independent depression (Fig. $6 C, E, F)$. The parameters of frequencyindependent depression were based on data from FS interneuron synapses onto MSNs. Hypothetical values of frequencydependent depression were derived from climbing fiber synapses onto Purkinje cells because they resemble FS synapses onto MSNs in terms of their basket-like morphology and dynamic range (Dittman and Regehr, 1998). In the case of frequencyindependent depression, sIPSCs acted like an attenuating filter, decreasing MSN firing rates similarly at all frequencies tested (Fig. $6 G, H)$. In contrast, sIPSCs displaying frequency-dependent inhibition acted like a high-pass filter because MSN firing rates were effectively inhibited at the low end of their dynamic range but not at the high (Fig. $6 G, H)$. Although these dynamic clamp experiments do not capture the precise timing or subcellular localization of excitatory and inhibitory inputs, they demonstrate the importance of frequency-independent depression at FS synapses to maintain effective inhibition of MSNs across a broad dynamic

range of inputs to the circuit.

\section{Feedforward inhibition is pathway specific}

The output of the striatum depends not only on the firing rates of MSNs, but also on which MSNs are firing. Approximately half of the MSNs in the striatum project to the GP and give rise to the indirect pathway while the other half project to the $\mathrm{SNr}$ and give rise to the direct pathway (Wilson, 1998). Despite the important distinctions between these pathways, there has never been an 
analysis of how feedforward inhibition affects one pathway relative to the other.

To distinguish direct- from indirectpathway MSNs in slices from Lhx6-GFP mice, Lhx6-GFP BAC transgenic mice were crossed to Drdla-tdTomato BAC transgenic mice (Shuen et al., 2008). The offspring of these crosses express tdTomato in direct-pathway MSNs and GFP in GABAergic interneurons, enabling the identification of direct-pathway MSNs, indirect-pathway MSNs, and GABAergic interneurons in a single brain slice (Fig. $7 A)$. We estimate that tdTomato expression in Drdla-tdTomato BAC transgenic mice labels $\sim 96 \%$ of direct-pathway MSNs (Table 2). This is in contrast to a previous report that suggested a larger number of unlabeled MSNs (Shuen et al., 2008), but is consistent with a second study that found more complete labeling in a similar mouse line (Gertler et al., 2008).

To determine whether feedforward inhibition is different onto direct- versus indirect-pathway MSNs, paired recordings were established between GFP+ FS interneurons and direct- or indirectpathway MSNs, identified using the presence or absence of tdTomato fluorescence (Fig. 7B). Over half (48/90) of FS $\rightarrow$ directpathway MSN pairs were functionally connected, compared with only $36 \%$ (27/ 77) of FS $\rightarrow$ indirect-pathway MSN pairs $(p=0.02)$ (Fig. 7C). It was often possible to sample multiple postsynaptic MSNs per presynaptic interneuron, enabling the connectivity of single FS interneurons with MSNs in both pathways to be assessed. Figure $7 B$ shows a recording from an FS interneuron that made inhibitory synapses onto MSNs in both pathways. Of 28 FS interneurons where synaptic connectivity was tested onto both direct- and indirectpathway MSNs, functional connections with both pathways were found for 11 neurons. This demonstrates that single FS interneurons can regulate the activity of both direct- and indirectpathway MSNs, but it does not rule out the possibility that some FS interneurons target MSNs of a single subtype.

Although the probability of synaptic connectivity was higher between FS interneurons and direct-pathway MSNs, inhibitory synapses onto MSNs in both pathways were similar (Fig. $7 D-G$ ). There was no difference in the uIPSC amplitude (501 $\pm 760 \mathrm{pA}$, $n=43$ vs $578 \pm 834 \mathrm{pA}, n=26 ; p=0.66)$, decay kinetics ( $8.1 \pm$ $3.6 \mathrm{~ms}$ vs $7.6 \pm 2.3 \mathrm{~ms} ; p=0.94), \operatorname{PPR}(0.69 \pm 0.25$ vs $0.66 \pm 0.18$; $p=0.62)$, latency $(0.84 \pm 0.17 \mathrm{~ms}$ vs $0.93 \pm 0.29 \mathrm{~ms} ; p=0.53)$ or short-term dynamics of FS synapses onto direct- or indirectpathway MSNs. Together, our data indicate that FS interneurons make similar synaptic contacts onto direct- and indirect-pathway MSNs, but show a significant preference for direct-pathway MSNs.

\section{Discussion}

Progress in understanding how striatal microcircuits control activity of MSNs has been hampered by difficulties in targeting
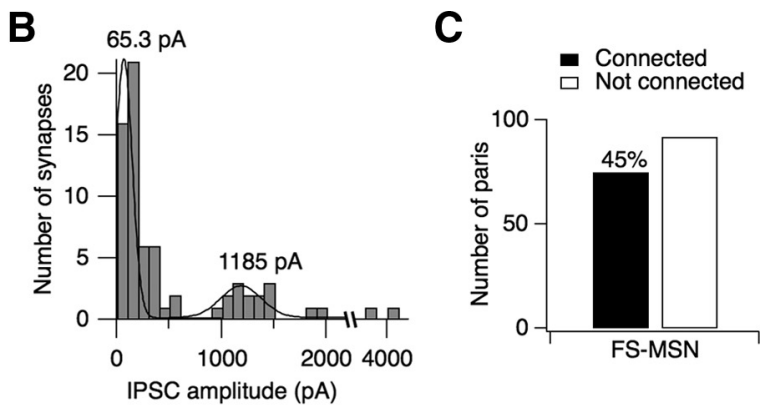

$\mathbf{E}$

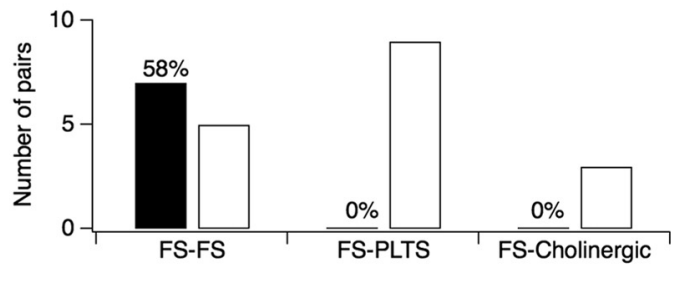

G

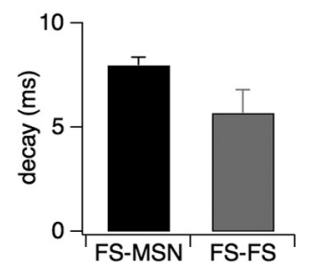

H

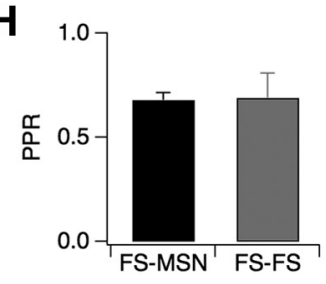

Figure 5. Local connectivity of FS interneurons. $A$, Paired recording showing a typical functional connection between a presynaptic FS interneuron and a postsynaptic MSN. B, Histogram showing the bimodal distribution of uIPSC amplitudes recorded

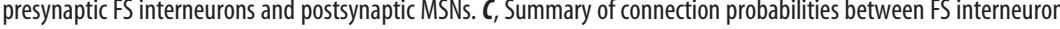
tween a pair of FS interneurons. $\boldsymbol{E}$, Summary of connection probabilities between FS interneurons and other interneuron types in highly connected to other FS interneurons (7/12) but not with PLTS interneurons (0/9) or cholinergic interneurons (0/3). All pairs were within a $250 \mu \mathrm{m}$ radius. $\boldsymbol{F}-\boldsymbol{H}$, Bar graphs comparing the conductances $(\boldsymbol{F})$, decay time constants $(\boldsymbol{G})$, and paired-pulse ratios $(\boldsymbol{H})$ of ulPSCs between FS-MSN and FS-FS pairs. Only the unitary conductance was significantly larger in FS-MSN pairs compared with that in FS-FS pairs $(p=0.009)$. ${ }^{*}$ Statistical significance $(p<0.05)$. Error bars, SEM.

GABAergic interneurons for electrophysiological analysis. Here we present the Lhx6-GFP BAC transgenic mouse line as a tool to rapidly identify GABAergic interneurons in living striatal slices. We find that FS interneurons represent the main source of feedforward inhibition in the mouse striatum and that their synapses are tuned for stable inhibition of MSNs across a broad frequency range. FS projections preferentially target direct-pathway MSNs relative to indirect-pathway MSNs, suggesting a mechanism for pathway-specific regulation of striatal output.

\section{GABAergic interneurons are labeled in the Lhx6-GFP BAC transgenic mice}

All neurons labeled with GFP in Lhx6-GFP mice have the electrophysiological properties of FS or PLTS interneurons, consistent with reports that these two cell types represent the majority of GABAergic interneurons in the striatum (Kawaguchi, 1993; Koós and Tepper, 1999; Tepper and Bolam, 2004). Although GFP expression in the Lhx6 line did not label all PV+ or NPY/SOM + neurons in the striatum, 95\% of GFP + neurons expressed one of these markers. Of the remaining $5 \%$ of GPF + neurons that did not express $\mathrm{PV}$ or NPY/SOM, 3\% likely represent CR-expressing interneurons. The remaining $2 \%$ may represent neurons in which interneuron markers were not detected due to low expression levels of the proteins or may represent small populations of 
A

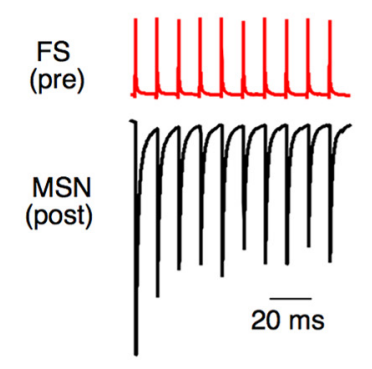

C

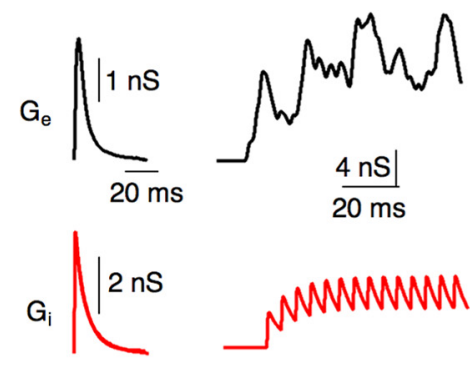

$20 \mathrm{~Hz}$

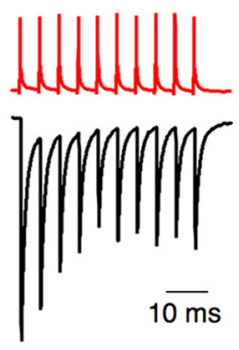

D
$50 \mathrm{~Hz}$

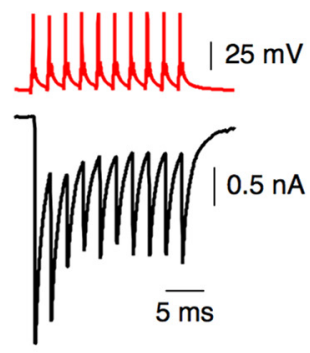

Excitation alone

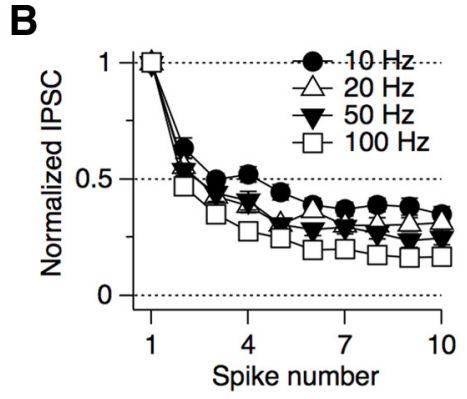

E

Excitation + inhibition

MSN

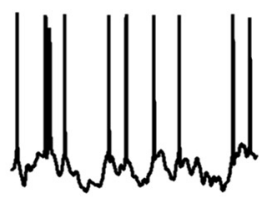

$25 \mathrm{mV}$
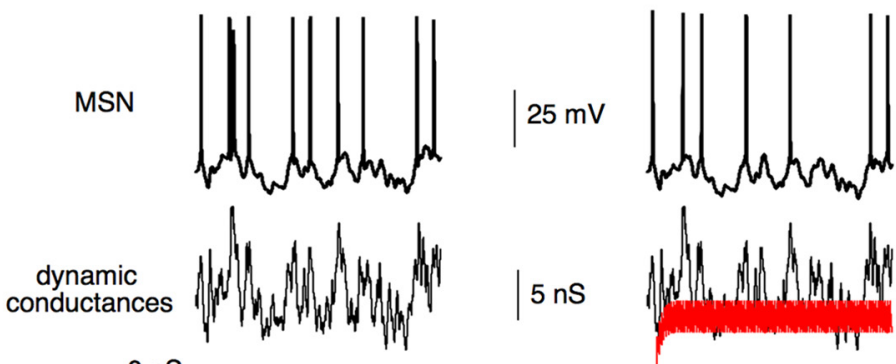

ons
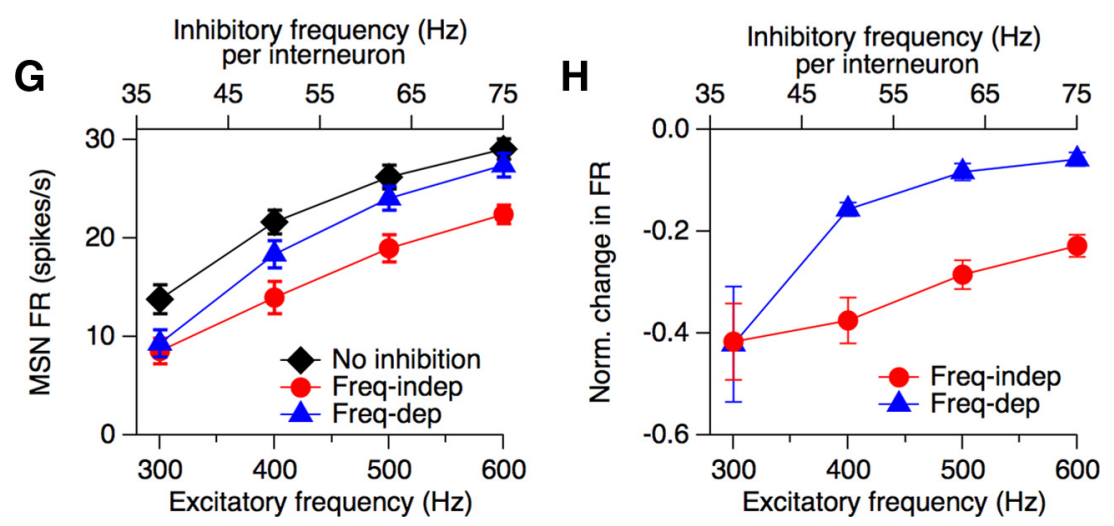

Figure 6. Short-term dynamics of synaptic transmission at FS-MSN synapses. A, Paired recording between a presynaptic FS interneuron and a postsynaptic MSN, showing typical short-term depression during a train of 10 action potentials at 10,20 , and $50 \mathrm{~Hz}$. B, Summary of synaptic depression of uIPSCs recorded in postsynaptic MSNs that accumulates during a train of 10 action potentials driven in presynaptic FS interneurons at 10,20,50, and $100 \mathrm{~Hz}(n=24)$. Depression is calculated as the average normalized value for IPSC $8-10$. Error bars, SEM. C, Top: Waveform of an individual sEPSC, scaled to a peak conductance of $2.3 \mathrm{nS}$ and a Poisson train of sEPSCs at an average frequency of $400 \mathrm{~Hz}$. Bottom: Waveform of an individual sIPSC, scaled to a peak conductance of $3.6 \mathrm{nS}$ and a train of SIPSCs at a constant frequency of $200 \mathrm{~Hz}$ (sum of 4 interneurons firing at $50 \mathrm{~Hz}$ ). D. Action potentials recorded in an MSN in response to a Poisson train of excitatory conductances at $400 \mathrm{~Hz}$. $\boldsymbol{E}$, Same as $\boldsymbol{D}$ but with the addition of $200 \mathrm{~Hz}$ inhibition (corresponding to 4 interneurons firing at $50 \mathrm{~Hz}$ each). $\boldsymbol{F}$, Theoretical levels of steady-state depression at different frequencies for inhibitory synapses showing frequency-independent depression (red trace) and frequency-dependent depression (blue trace). The boxed region shows the range of frequencies ( $35-75 \mathrm{~Hz}$ ) used for the experiment in $\boldsymbol{G}$ and $\boldsymbol{H}$. $\boldsymbol{G}$, Average input-output curves recorded from MSNs $(n=15)$ without inhibition (black trace) or in conjunction with inhibition showing frequency-independent (red trace) or frequency-dependent depression (blue trace). Excitatory and inhibitory frequencies were adjusted proportionately to maintain a 2:1 (E:I) frequency ratio. Excitatory frequencies ranged from 300 to $600 \mathrm{~Hz}$ and excitatory conductance was kept constant at $2.3 \mathrm{nS}$. Inhibitory frequencies ranged from 37.5 to $75 \mathrm{~Hz}$ per interneuron (total frequency $=150-300 \mathrm{~Hz}$ ) and inhibitory conductances were scaled by the predicted level of steady-state depression $(\boldsymbol{F})$. $\boldsymbol{H}$, Normalized change in MSN firing rate at each frequency tested, due to the addition of frequency-independent or frequencydependent depression. Note that frequency-independent depression enables robust inhibition across the dynamic range tested, but frequency-dependent depression is less effective at high frequencies.

other classes of interneurons, such as VIP or AADC-expressing interneurons (Theriault and Landis, 1987; Mura et al., 2000).

A major subpopulation of GABAergic interneurons that express CR in rats was notably absent from the striatum of mice, indicating an important species distinction in the prevalence of CR interneurons (Wu and Parent, 2000). In rats, CR+ interneurons may correspond electrophysiologically to low-threshold spiking (LTS) interneurons that lack plateau potentials and provide feedforward inhibition to MSNs, similar in magnitude to that from FS interneurons (Koós and Tepper, 1999; Tepper and Bolam, 2004). These LTS interneurons were never encountered in the mouse striatum.

\section{Implications for different excitatory synapses onto FS and}

\section{PLTS interneurons}

FS interneurons receive significantly larger amplitude excitatory inputs than PLTS interneurons. This could mean that FS interneurons are more sensitive to changes in excitatory inputs or it could reflect adaptation of synaptic strength to drive action potentials. FS interneurons have a lower membrane input resistance and thus require stronger excitatory currents to drive them to spike threshold compared with PLTS interneurons, which exhibit higher input resistances. Support for the latter hypothesis comes from our observation that even small EPSPs are sufficient to drive firing in PLTS interneurons (data not shown). 
We found that FS and PLTS interneurons express different types of synaptic AMPA receptors. The $I-V$ curve of the AMPA component was strongly rectifying in FS interneurons but only weakly rectifying in PLTS interneurons, suggesting a much stronger contribution from GluR2lacking AMPA receptors in FS interneurons relative to that in PLTS interneurons. The presence of GluR2-lacking, $\mathrm{Ca}^{2+}$ permeable AMPARs is a common feature of excitatory synapses onto FS interneurons in other brain regions as well, where they are hypothesized to endow FS interneurons with greater temporal precision and unique forms of short- and long-term plasticity (McBain and Fisahn, 2001; Isaac et al., 2007). The modest rectification and slower decay time constants of AMPA receptor-mediated currents in PLTS interneurons suggests the presence of both GluR2lacking and GluR2-containing AMPAR, similar to AMPAR expressed in MSNs (Kreitzer and Malenka, 2007). In summary, differences in the glutamate receptors of FS and PLTS interneurons may endow these cell types with unique features, including differential recruitment in the striatal microcircuit, distinct firing patterns during synaptic activation, and cell-type-specific forms of neuromodulation and synaptic plasticity.

\section{Alternative roles for PLTS interneurons in regulating striatal function}

Although PLTS interneurons receive excitatory inputs, our results demonstrate that they are sparsely connected to other neurons in the striatal circuit and form weak inhibitory synapses, likely onto the distal dendrites of MSNs. This is consistent with anatomical observations that the axonal arborizations of PLTS interneurons are far less dense compared with those of FS interneurons (Kawaguchi, 1993), and that SOM+ GABAergic terminals have been observed on the dendrites of MSNs (Kubota and Kawaguchi, 2000). This suggests that synapses from PLTS interneurons may affect dendritic excitability but are unlikely to directly influence MSN output. Furthermore, although the apparent connectivity of PLTS interneurons is low, we cannot rule out that these connections are strengthened under certain conditions such as strong neuromodulation or in disease states (Dehorter et al., 2009).

Rather than playing a role in feedforward inhibition, the main function of PLTS interneurons may instead be to release neuromodulators such as NPY, SOM, and NO, which could modulate striatal circuitry with distinct spatial and temporal patterns relative to fast synaptic transmission (Steinert et al., 2008; O'Donnell and Grace, 1997; West et al., 2002; West and Grace, 2004; Galarraga et al., 2007; Lopez-Huerta et al., 2008). Axon terminals containing only NPY and no GABA have also been observed at synapses onto MSNs (Aoki and Pickel, 1989) and in close proximity to glia

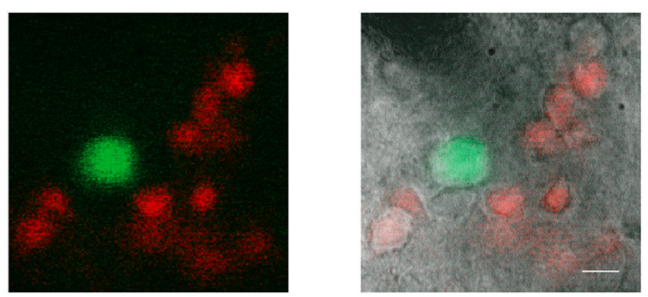

C

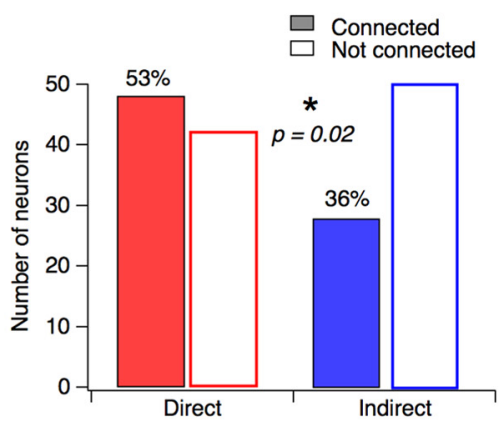

E

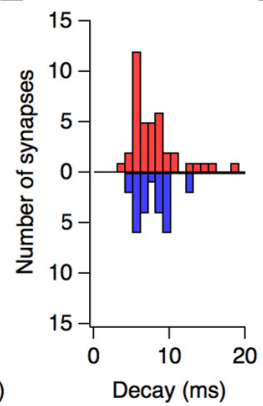

$\mathbf{F}$

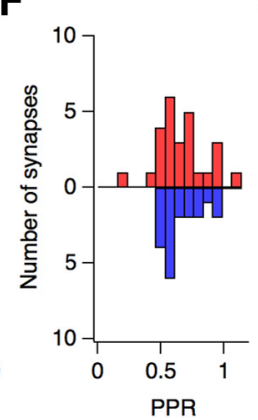

G

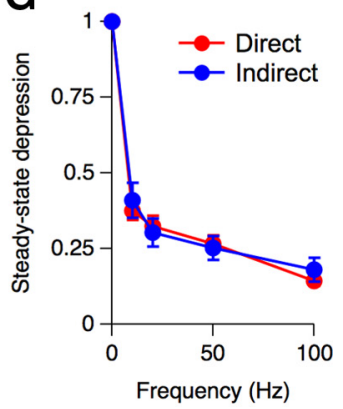

Figure 7. FS interneurons preferentially target direct-pathway MSNs. A, IR-DIC (left) and fluorescent images (middle) from a entification of GFP + interneurons, direct-pathway MSNs (tdTomato +), and indirect-pathway MSNs (unlabeled) in the slice. functional connections with both a direct- and an indirect-pathway MSN. C, Summary of connectivity measured with paired

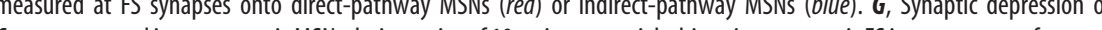
PSC $S_{8-10}$ measured in postsynaptic MSNs during trains of 10 action potentials driven in presynaptic FS interneurons at frequencies of 10,20,50, and $100 \mathrm{~Hz}$. Similar frequency-independent depression was observed at synapses onto both direct $(n=16)$ - and indirect $(n=8)$-pathway MSNs. Error bars, SEM.

and blood vessels (Aoki and Pickel, 1990), suggesting that PLTS interneurons may potentially regulate blood flow or glial signaling in the striatum.

\section{Dynamics of feedforward inhibition influences input-output transformations of MSNs}

Although previous studies have identified FS interneurons as a source of feedforward inhibition capable of modulating MSN firing rates at low frequencies (Koós and Tepper, 1999; Koós et al., 2004), few studies have considered how they operate over a range of dynamic inputs to the circuit that would be experienced in vivo. MSNs receive thousands of excitatory inputs $(10,000-$ 15,000 ) (Wilson, 1998) but receive inhibitory inputs from only 4-27 FS interneurons (Koós and Tepper, 1999). Despite this asymmetry, recordings from cultured MSNs suggest that excitation and inhibition remain balanced across a broad range of inputs (Blackwell et al., 2003). This suggests that in the striatum, 
inhibition from a small number of FS interneurons balances excitation from thousands of excitatory inputs, a neuronal architecture likely preserved in a number of other brain regions.

How could inhibition from so few synapses balance excitation from so many? Individual cortical pyramidal neurons rarely fire $>10 \mathrm{~Hz}$ (de Kock et al., 2007), but FS interneurons exhibit firing rates an order of magnitude faster, typically firing $10-100 \mathrm{~Hz}$ in vivo (Berke et al., 2004; Berke, 2008) and are more responsive to excitatory inputs than MSNs (Parthasarathy and Graybiel, 1997; Ramanathan et al., 2002; Mallet et al., 2005). This difference in operating frequency could permit a small number of rapidly firing inhibitory inputs to balance a large number of slowly firing excitatory inputs. This scenario requires that FS synapses maintain the same level of synaptic efficacy over a broader dynamic range than excitatory synapses. This capability is mediated by the short-term dynamics of FS synapses, which depress during repetitive activation in a manner that is relatively independent of frequency. This frequency-independent depression has been observed at a number of other synapses (Galarreta and Hestrin, 1998; Varela et al., 1999; Telgkamp and Raman, 2002; Gabernet et al., 2005; Arenz et al., 2008) and can enable linear computations of circuit input (Bagnall et al., 2008). In the striatum, frequencyindependent depression acts as an attenuating filter, preserving the relative input-output computations of MSNs. If instead, depression at FS synapses was not frequency-independent and changed in a frequency-dependent manner, it would perform a high-pass filtering function on the outputs of MSNs, altering the nature of striatal processing.

\section{Implications of different feedforward inhibition onto direct- and indirect-pathway MSNs for striatal function}

In this study, we show for the first time that FS interneurons target both direct- and indirect-pathway MSNs, but are more likely to target MSNs in the direct pathway. Although the direct and indirect pathways appear to serve distinct functions, nothing is known about the computational principles that distinguish these circuits. One early hint that cortical inputs might differentially affect striatal output pathways came from a study of immediate-early gene activation following sensorimotor cortical stimulation (Parthasarathy and Graybiel, 1997). In this work, we observed that cortical stimulation led to the selective activation of PV+ (fast-spiking) interneurons and enkephalinpositive (indirect-pathway) MSNs, but far fewer direct-pathway MSNs. Our finding that feedforward inhibition is significantly more prominent in striatal direct-pathway MSNs provides a mechanism that could underlie their relatively weaker activation following cortical stimulation.

The finding that feedforward inhibition is a more prominent feature of the direct-pathway may underlie the fact that neurological diseases associated with hyper-activation of the directpathway (Tourette syndrome and dystonia) are correlated with decreased numbers of striatal FS interneurons (Gernert et al., 2000; Kalanithi et al., 2005). The ability of FS interneurons to target hundreds of MSNs and differentially regulate direct- and indirect-pathway MSNs places them in a central position to regulate the balance of striatal output. By targeting a relatively small population of neurons in the striatum, it may be possible to therapeutically adjust the balance of direct- and indirect-pathway striatal output to alleviate motor dysfunction in a number of neurological diseases associated with the basal ganglia, including Tourette syndrome, dystonia, Parkinson disease, and Huntington's disease.

\section{References}

Abbott LF, Varela JA, Sen K, Nelson SB (1997) Synaptic depression and cortical gain control. Science 275:220-224.

Aoki C, Pickel VM (1989) Neuropeptide Y in the cerebral cortex and the caudate-putamen nuclei: ultrastructural basis for interactions with GABAergic and non-GABAergic neurons. J Neurosci 9:4333-4354.

Aoki C, Pickel VM (1990) Neuropeptide Y in cortex and striatum. Ultrastructural distribution and coexistence with classical neurotransmitters and neuropeptides. Ann N Y Acad Sci 611:186-205.

Arenz A, Silver RA, Schaefer AT, Margrie TW (2008) The contribution of single synapses to sensory representation in vivo. Science 321:977-980.

Bagnall MW, McElvain LE, Faulstich M, du Lac S (2008) Frequencyindependent synaptic transmission supports a linear vestibular behavior. Neuron 60:343-352.

Berke JD (2008) Uncoordinated firing rate changes of striatal fast-spiking interneurons during behavioral task performance. J Neurosci 28:1007510080.

Berke JD, Okatan M, Skurski J, Eichenbaum HB (2004) Oscillatory entrainment of striatal neurons in freely moving rats. Neuron 43:883-896.

Blackwell KT, Czubayko U, Plenz D (2003) Quantitative estimate of synaptic inputs to striatal neurons during up and down states in vitro. J Neurosci 23:9123-9132.

Cobos I, Long JE, Thwin MT, Rubenstein JL (2006) Cellular patterns of transcription factor expression in developing cortical interneurons. Cereb Cortex 16 (Suppl 1):i82-i88.

Dehorter N, Guigoni C, Lopez C, Hirsch J, Eusebio A, Ben-Ari Y, Hammond C (2009) Dopamine-deprived striatal GABAergic interneurons burst and generate repetitive gigantic IPSCs in medium spiny neurons. J Neurosci 29:7776-7787.

de Kock CP, Bruno RM, Spors H, Sakmann B (2007) Layer- and cell-typespecific suprathreshold stimulus representation in rat primary somatosensory cortex. J Physiol 581:139-154.

Dittman JS, Regehr WG (1998) Calcium dependence and recovery kinetics of presynaptic depression at the climbing fiber to Purkinje cell synapse. J Neurosci 18:6147-6162.

Figueredo-Cardenas G, Medina L, Reiner A (1996) Calretinin is largely localized to a unique population of striatal interneurons in rats. Brain Res 709:145-150.

Földy C, Lee SY, Szabadics J, Neu A, Soltesz I (2007) Cell type-specific gating of perisomatic inhibition by cholecystokinin. Nat Neurosci 10:11281130 .

Gabernet L, Jadhav SP, Feldman DE, Carandini M, Scanziani M (2005) Somatosensory integration controlled by dynamic thalamocortical feedforward inhibition. Neuron 48:315-327.

Galarraga E, Vilchis C, Tkatch T, Salgado H, Tecuapetla F, Perez-Rosello T, Perez-Garci E, Hernandez-Echeagaray E, Surmeier DJ, Bargas J (2007) Somatostatinergic modulation of firing pattern and calcium-activated potassium currents in medium spiny neostriatal neurons. Neuroscience 146:537-554.

Galarreta M, Hestrin S (1998) Frequency-dependent synaptic depression and the balance of excitation and inhibition in the neocortex. Nat Neurosci 1:587-594.

Galvan A, Wichmann T (2007) GABAergic circuits in the basal ganglia and movement disorders. Prog Brain Res 160:287-312.

Gernert M, Hamann M, Bennay M, Löscher W, Richter A (2000) Deficit of striatal parvalbumin-reactive GABAergic interneurons and decreased basal ganglia output in a genetic rodent model of idiopathic paroxysmal dystonia. J Neurosci 20:7052-7058.

Gertler TS, Chan CS, Surmeier DJ (2008) Dichotomous anatomical properties of adult striatal medium spiny neurons. J Neurosci 28:1081410824.

Graybiel AM, Canales JJ, Capper-Loup C (2000) Levodopa-induced dyskinesias and dopamine-dependent stereotypies: a new hypothesis. Trends Neurosci 23:S71-77.

Isaac JT, Ashby M, McBain CJ (2007) The role of the GluR2 subunit in AMPA receptor function and synaptic plasticity. Neuron 54:859-871.

Kalanithi PS, Zheng W, Kataoka Y, DiFiglia M, Grantz H, Saper CB, Schwartz ML, Leckman JF, Vaccarino FM (2005) Altered parvalbumin-positive neuron distribution in basal ganglia of individuals with Tourette syndrome. Proc Natl Acad Sci U S A 102:13307-13312.

Kawaguchi Y (1993) Physiological, morphological, and histochemical char- 
acterization of three classes of interneurons in rat neostriatum. J Neurosci 13:4908-4923.

Kawaguchi Y, Wilson CJ, Augood SJ, Emson PC (1995) Striatal interneurones: chemical, physiological and morphological characterization. Trends Neurosci 18:527-535.

Koós T, Tepper JM (1999) Inhibitory control of neostriatal projection neurons by GABAergic interneurons. Nat Neurosci 2:467-472.

Koos T, Tepper JM, Wilson CJ (2004) Comparison of IPSCs evoked by spiny and fast-spiking neurons in the neostriatum. J Neurosci 24:79167922.

Kreitzer AC (2009) Physiology and pharmacology of striatal neurons. Annu Rev Neurosci 32:127-147.

Kreitzer AC, Malenka RC (2007) Endocannabinoid-mediated rescue of striatal LTD and motor deficits in Parkinson's disease models. Nature 445:643-647.

Kreitzer AC, Malenka RC (2008) Striatal plasticity and basal ganglia circuit function. Neuron 60:543-554.

Kubota Y, Kawaguchi Y (2000) Dependence of GABAergic synaptic areas on the interneuron type and target size. J Neurosci 20:375-386.

Lopez-Huerta VG, Tecuapetla F, Guzman JN, Bargas J, Galarraga E (2008) Presynaptic modulation by somatostatin in the neostriatum. Neurochem Res 33:1452-1458.

Mallet N, Le Moine C, Charpier S, Gonon F (2005) Feedforward inhibition of projection neurons by fast-spiking GABA interneurons in the rat striatum in vivo. J Neurosci 25:3857-3869.

Mallet N, Ballion B, Le Moine C, Gonon F (2006) Cortical inputs and GABA interneurons imbalance projection neurons in the striatum of parkinsonian rats. J Neurosci 26:3875-3884.

Marin O, Anderson SA, Rubenstein JL (2000) Origin and molecular specification of striatal interneurons. J Neurosci 20:6063-6076.

Markram H, Gupta A, Uziel A, Wang Y, Tsodyks M (1998) Information processing with frequency-dependent synaptic connections. Neurobiol Learn Mem 70:101-112.

McBain CJ, Fisahn A (2001) Interneurons unbound. Nat Rev Neurosci 2:11-23.

Mura A, Linder JC, Young SJ, Groves PM (2000) Striatal cells containing aromatic L-amino acid decarboxylase: an immunohistochemical comparison with other classes of striatal neurons. Neuroscience 98:501-511.

Nisenbaum ES, Berger TW (1992) Functionally distinct subpopulations of striatal neurons are differentially regulated by GABAergic and dopaminergic inputs-I. In vivo analysis. Neuroscience 48:561-578.

O'Donnell P, Grace AA (1997) Cortical afferents modulate striatal gap junction permeability via nitric oxide. Neuroscience 76:1-5.

Palop JJ, Chin J, Bien-Ly N, Massaro C, Yeung BZ, Yu GQ, Mucke L (2005) Vulnerability of dentate granule cells to disruption of arc expression in human amyloid precursor protein transgenic mice. J Neurosci 25:96869693.
Parthasarathy HB, Graybiel AM (1997) Cortically driven immediate-early gene expression reflects modular influence of sensorimotor cortex on identified striatal neurons in the squirrel monkey. J Neurosci 17:24772491.

Ramanathan S, Hanley JJ, Deniau JM, Bolam JP (2002) Synaptic convergence of motor and somatosensory cortical afferents onto GABAergic interneurons in the rat striatum. J Neurosci 22:8158-8169.

Shuen JA, Chen M, Gloss B, Calakos N (2008) Drd1a-tdTomato BAC transgenic mice for simultaneous visualization of medium spiny neurons in the direct and indirect pathways of the basal ganglia. J Neurosci 28:26812685.

Smith Y, Bevan MD, Shink E, Bolam JP (1998) Microcircuitry of the direct and indirect pathways of the basal ganglia. Neuroscience 86:353-387.

Steinert JR, Kopp-Scheinpflug C, Baker C, Challiss RA, Mistry R, Haustein MD, Griffin SJ, Tong H, Graham BP, Forsythe ID (2008) Nitric oxide is a volume transmitter regulating postsynaptic excitability at a glutamatergic synapse. Neuron 60:642-656.

Taverna S, Canciani B, Pennartz CM (2007) Membrane properties and synaptic connectivity of fast-spiking interneurons in rat ventral striatum. Brain Res 1152:49-56.

Telgkamp P, Raman IM (2002) Depression of inhibitory synaptic transmission between Purkinje cells and neurons of the cerebellar nuclei. J Neurosci 22:8447-8457.

Tepper JM, Bolam JP (2004) Functional diversity and specificity of neostriatal interneurons. Curr Opin Neurobiol 14:685-692.

Tepper JM, Koós T, Wilson CJ (2004) GABAergic microcircuits in the neostriatum. Trends Neurosci 27:662-669.

Theriault E, Landis DM (1987) Morphology of striatal neurons containing VIP-like immunoreactivity. J Comp Neurol 256:1-13.

Varela JA, Song S, Turrigiano GG, Nelson SB (1999) Differential depression at excitatory and inhibitory synapses in visual cortex. J Neurosci 19:4293-4304.

West AR, Grace AA (2004) The nitric oxide-guanylyl cyclase signaling pathway modulates membrane activity States and electrophysiological properties of striatal medium spiny neurons recorded in vivo. J Neurosci 24:1924-1935.

West AR, Galloway MP, Grace AA (2002) Regulation of striatal dopamine neurotransmission by nitric oxide: effector pathways and signaling mechanisms. Synapse 44:227-245.

Wilson CJ (1998) The synaptic organization of the brain, Ed 4. Oxford: Oxford Scholarship.

Wilson CJ (2007) GABAergic inhibition in the neostriatum. Prog Brain Res 160:91-110.

Wu Y, Parent A (2000) Striatal interneurons expressing calretinin, parvalbumin or NADPH-diaphorase: a comparative study in the rat, monkey and human. Brain Res 863:182-191. 